June 1994

\title{
$\zeta$-function calculation of the Weyl determinant for two-dimensional non-abelian gauge theories in a curved background and its W-Z-W terms
}

\author{
Luca Griguolo \\ International School for Advanced Studies \\ via Beirut 2, 34100 Trieste, Italy \\ I.N.F.N. Sezione di Trieste
}

\begin{abstract}
Using a cohomological characterization of the consistent and the covariant Lorentz and gauge anomalies, derived from the complexification of the relevant algebras, we study in $d=2$ the definition of the Weyl determinant for a non-abelian theory with Riemannian background. We obtain two second order operators that produce, by means of $\zeta$-function regularization, respectively the consistent and the covariant Lorentz and gauge anomalies, preserving diffeomorphism invariance. We compute exactly their functional determinants and the W-Z-W terms: the "consistent" determinant agrees with the non-abelian generalization of the classical Leutwyler's result, while the "covariant" one gives rise to a covariant version of the usual Wess-Zumino-Witten action.
\end{abstract}




\section{Introduction}

In the first eighties it was realized that a rich algebraic and geometrical structure subtends the existence of consistent anomalies: their characterization as solutions of a cohomological problem [ [] and its relation with deep algebraic geometrical theorems, as the index theorem [2, 3], were crucial tools in understanding interesting phenomena.

A complementary approach to the problem of the anomaly relies on the construction of some representations of the anomaly algebra: with regard to theories describing gauge or gravitational interactions of spin one-half fermions, this is realized as the determinant of a relevant Weyl operator.

On a compact Riemannian manifold the eigenvalue problem for this operator is not well defined and the local anomaly is just the manifestation of this fact: the determinant can be characterized, from a geometrical point of view, as a line-bundle over the space of the gauge orbit [2]. The non-triviality of this line-bundle, linked to the non-vanishing of the anomaly, makes impossible to have a global section i.e. to have gauge invariance for the determinant.

In an analytical approach, where one essentially tries to give a meaning to an infinite product of eigenvalues, the problem appears from the very beginning, any possible definition of the eigenvalues of the Weyl operator being ambiguos by a phase factor [3]. For a gauge theory with connections on trivial principal-bundle, one can easily by-pass the problem working with a Dirac operator, in which the gauge fields are coupled only to the left or the right component of the spinor. $\zeta$-function regularization [4, 5] provides a well-defined procedure to define the determinant of operators of this type, reproducing the perturbative and cohomological results for the consistent local anomaly; in the gravitational case this trick does not work, due to the crucial presence of the n-bein fields that couple to both components. An analytical definition and an explicit computation of the chiral determinant in curved space appeared in [6, 7], using a completely different approach based on integrating polynomials and a regularization of the fermion propagator.

In reference [8] we have developed a systematic way to define the Weyl determinant for gauge theories, based on its $\zeta$-function definition in terms of second order operators. The complexification of the gauge group was the main tool in this approach; we have shown how the related cohomological problem for the anomaly has in this case two different solutions, one giving the consistent anomaly and the other one generating the covariant anomaly. The correct operators were obtained requiring that the gauge variations of their functional determinants, in this generalized setting, realize the consistent solution. In this way we have also clarified the structure of the covariant anomalies and their relation with functional determinant techniques.

In this paper we compute the determinant for a two-dimensional non-abelian chiral gauge theory in a curved Riemannian background: the complexification of the gauge groups (color $S U(N)$ and local-frame $S O(2, R)$ ) can be avoided, simplifying the analytical calculations, and a direct comparison of our formalism with previous results [7] is possible. We could also directly test the properties of the covariant solution and indeed we give the explicit form of 
the covariant W-Z-W term [9], obtained from an extended $S L(2, C)$ transformation on the functional determinant.

The paper is organized as follows: to make it self-contained we describe in Sect.2 the extended cohomological problem in $d=2 n$ dimension; in Sect. 3 we turn our attention to $d=2$, writing the anomalies as derived from the descent equations and explaining the operatorial approach; in Sect. 4 and Sect.5 we find the correct second order operators linked to the covariant and consistent anomaly, respectively, and we compute their determinants and the related W-Z-W terms.

\section{The extended cohomological problem and its solution}

The classical action for a chiral spinor coupled to a gauge connection on a Riemannian manifold $M$ of dimension $2 n$ is:

$$
\begin{aligned}
S_{c l} & =\int d^{2 n} x \sqrt{g} \bar{\psi} D \psi, \\
D & =e_{a}^{\mu} \sigma_{a} i\left(\partial_{\mu}+i A_{\mu}+\frac{1}{4} \Omega_{\mu c d} \tilde{\sigma}_{c} \sigma_{d}\right) .
\end{aligned}
$$

We have used the canonical volume form of $M$

$$
d^{2 n} x \sqrt{g}
$$

$g_{\mu \nu}$ being a fixed metric. $E_{\mu a}$ are the $2 n$-bein fields (with inverse $e_{a}^{\mu}$ ) and $\Omega_{\mu c d}$ is the spinconnection linked to the metric tensor

$$
\begin{aligned}
g_{\mu \nu} & =E_{\mu a} E_{\nu a} \\
\Omega_{\mu a b} & =e_{a}^{\nu}\left(\partial_{\mu} E_{\nu b}-\Gamma_{\mu \nu}^{\lambda} E_{\lambda b}\right) .
\end{aligned}
$$

We assume the absence of torsion, so $\Gamma_{\mu \nu}^{\lambda}$ is the usual Levi-Civita connection.

$$
A_{\mu}=A_{\mu}^{a} T_{a}
$$

is a gauge connection belonging to a (trivial) $S U(N)$ principal bundle over $M, T_{a}$ being a basis for the $S U(N)$ Lie algebra:

$$
\begin{aligned}
{\left[T_{a}, T_{b}\right] } & =i f_{a b c} T_{c} \\
\operatorname{tr}\left(T_{a} T_{b}\right) & =\frac{1}{2} \delta_{a b}
\end{aligned}
$$

The Weyl matrices $\sigma_{a}$ represent the algebra

$$
\sigma_{a} \tilde{\sigma}_{b}+\sigma_{b} \tilde{\sigma}_{a}=2 \delta_{a b}
$$

$\tilde{\sigma}_{a}$ being the other inequivalent representation, existing for $d=2 n$ [10. 
The vacuum functional of chiral fermions on this backgrounds is formally defined as

$$
\Gamma[A, E]=-\log \operatorname{det}[D] .
$$

Immediately we are faced with a basic difficulty in defining the determinant for the Weyl operator $D$ : it maps a chiral spinor on a spinor of opposite chirality

$$
D: \Gamma\left(S_{+}\right)_{M} \rightarrow \Gamma\left(S_{-}\right)_{M}
$$

where $\Gamma\left(S_{+}\right)_{M}\left(\Gamma\left(S_{-}\right)_{M}\right)$ is the space of right (left) sections of the vector bundle associated by the Dirac representation to the spin-principal bundle on $M . \Gamma\left(S_{+}\right)_{M}$ and $\Gamma\left(S_{-}\right)_{M}$ are different Hilbert spaces and there is no canonical isomorphism between them: $D$ does not map a Hilbert space into itself and we have no canonical way to define a meaningful eigenvalue problem, that is essential for the construction of the determinant. A way out is to try to define the Weyl determinant by means of an operator with a good eigenvalue problem but, in general, some of the classical properties are lost after this substitution. In particular the covariance properties of $D$ could be destroyed, breaking the naive invariance of eq.(6) under gauge, diffeomorphism and local Lorentz transformations.

Disregarding problems of globality (namely assuming that $M$ is parallelizable), the invariance properties of eq.(6) derived from the symmetry of the classical action eq.(11), are:

(i) local gauge invariance (local $S U(N)$ transformations)

$$
\begin{aligned}
\delta^{G}(\lambda) A_{\mu}^{a} & =\partial_{\mu} \lambda^{a}+i\left[A_{\mu}, \lambda\right]^{a}, \\
\delta^{G}(\lambda) E_{\mu a} & =0,
\end{aligned}
$$

where we have defined $\lambda=\lambda_{a} T_{a}$;

(ii) local Lorentz invariance (local $S O(2 n, R)$ transformations)

$$
\begin{aligned}
\delta^{L}(\tau) A_{\mu}^{a} & =0 \\
\delta^{L}(\tau) E_{\mu a} & =\tau_{a b} E_{\mu b}
\end{aligned}
$$

where we have defined $\tau_{a b}=-\tau_{b a}, \tau=\frac{1}{4} \tau_{a b} \tilde{\sigma}_{a} \sigma_{b}$;

(iii) diffeomorphism invariance (local $G L(2 n, R)$ tranformations)

$$
\begin{aligned}
\delta^{D}(\xi) A_{\mu}^{a} & =\partial_{\mu} \xi^{\nu} A_{\nu}^{a}+\partial_{\nu} A_{\mu}^{a} \xi^{\nu} \\
\delta^{D}(\xi) E_{\mu a} & =\partial_{\mu} \xi^{\nu} E_{\nu a}+\partial_{\nu} E_{\mu a} \xi^{\nu} .
\end{aligned}
$$

If we want to describe these symmetries in the more general case of non-trivial principal bundles and non parallelizable manifolds, we have to introduce fixed background connections $A_{\mu}^{0}$ and $\Omega_{\mu a b}^{0}$ in order to get a global description on the base manifold [9, 11].

The algebra of $\delta^{G}, \delta^{L}$ and $\delta^{D}$ is:

$$
\begin{aligned}
{\left[\delta^{G}\left(\lambda_{1}\right), \delta^{G}\left(\lambda_{2}\right)\right] } & =\delta^{G}\left(\left[\lambda_{1}, \lambda_{2}\right]\right), \\
{\left[\delta^{D}\left(\xi_{1}\right), \delta^{D}\left(\xi_{2}\right)\right] } & =\delta^{D}\left(\left[\xi_{1}, \xi_{2}\right]_{L}\right), \\
{\left[\delta^{L}\left(\tau_{1}\right), \delta^{L}\left(\tau_{2}\right)\right] } & =\delta^{L}\left(\left[\tau_{1}, \tau_{2}\right]\right), \\
{\left[\delta^{D}(\xi), \delta^{L}(\tau)\right] } & =\delta^{L}\left(\xi^{\mu} D_{\mu} \tau\right),
\end{aligned}
$$


where

$$
\left[\xi_{1}, \xi_{2}\right]_{L}^{\mu}=\xi_{1}^{\nu} \partial_{\nu} \xi_{2}^{\mu}-\xi_{2}^{\nu} \partial_{\nu} \xi_{1}^{\mu}
$$

and $D_{\mu}$ is the covariant derivative on $S O(2 n, R)$; all the other commutators vanish.

If we could be able to construct $\Gamma[A, E]$ preserving the classical properties of the Weyl operator, the invariance of the action eq.(11) under the just described symmetries would have been translated into

$$
\delta^{G}(\lambda) \Gamma=\delta^{L}(\tau) \Gamma=\delta^{D}(\xi) \Gamma=0 .
$$

A meaningful definition of the vacuum functional, in general, will modify eq.(12), generating anomalies:

$$
\begin{aligned}
\delta^{G}(\lambda) \Gamma[A, E] & =a^{G}(\lambda), \\
\delta^{L}(\tau) \Gamma[A, E] & =a^{L}(\tau), \\
\delta^{D}(\xi) \Gamma[A, E] & =a^{D}(\xi) .
\end{aligned}
$$

The algebra eq.(11) gives strong constraints on the right-hand side of eqs.(13), the socalled Wess-Zumino consistency conditions:

$$
\begin{aligned}
\delta^{G}\left(\lambda_{1}\right) a^{G}\left(\lambda_{2}\right)-\delta^{G}\left(\lambda_{2}\right) a^{G}\left(\lambda_{1}\right) & =a^{G}\left(\left[\lambda_{1}, \lambda_{2}\right]\right), \\
\delta^{L}\left(\tau_{1}\right) a^{L}\left(\tau_{2}\right)-\delta^{L}\left(\tau_{2}\right) a^{L}\left(\tau_{1}\right) & =a^{L}\left(\left[\tau_{1}, \tau_{2}\right]\right), \\
\delta^{D}\left(\xi_{1}\right) a^{D}\left(\xi_{2}\right)-\delta^{D}\left(\xi_{2}\right) a^{D}\left(\xi_{1}\right) & =a^{D}\left(\left[\xi_{1}, \xi_{2}\right]_{L}\right), \\
\delta^{D}(\xi) a^{L}(\tau)-\delta^{L}(\tau) a^{D}(\xi) & =a^{L}\left(\xi^{\mu} D_{\mu} \tau\right),
\end{aligned}
$$

that are equivalent, after having turned the Lie algebra valued parameters $\lambda, \tau$ and $\xi$ into anticommuting ghosts and having defined the action of $\delta^{G, L, D}$, on them, to a cohomological problem [1]. The information encoded in eqs.(14) is enough to obtain, up to a global coefficient, the general form of $a^{G}, a^{L}$ and $a^{D}$, requiring their locality on the fields and their derivatives. We remark that the cohomological solutions naturally implement the arbitrariness of the regularization procedure on the computation of $\Gamma[A, E]: a^{G, L, D}$ are obtained up to coboundaries of the relevant B.R.S.T. operators that correspond to the addition of suitables local terms on the right-hand side of eq.(6).

The solutions of eqs.(14) are called consistent anomalies and a correct definition of the Weyl determinant must fullfil them, according eq.(13). In the following we assume $a^{D}(\xi)=0$ because we are interested in a diffeomorphism invariant definition of $\Gamma[A, E]$; this choice is compatible with eqs.(14) (for parallelizable manifolds) and it can be always achieved by adding to the vacuum functional a suitable local (but non-polynomial) Wess-Zumino term on the $2 n$-bein fields 112.

If we extend $S U(N)$ to $S L(N, C)$ and $S O(2 n, R)$ to $S O(2 n, C)$ we can derive from eqs.(14) not only the consistent anomalies but also the covariant anomalies [8, 13]. Let us take for example $\delta^{G}$ : we make the decomposition

$$
\delta^{G}(\lambda)=\delta_{1}^{G}(\lambda)+\delta_{2}^{G}(\lambda)
$$


with $\delta_{1}^{G}(\lambda)$ generating gauge transformations of $S U(N)$ type (now seen as subgroup of $S L(N, C)$ ) and $\delta_{2}^{G}(\lambda)$ acting on the ortogonal invariant complement. The algebra of $\delta_{1}^{G}$ and $\delta_{2}^{G}$ is:

$$
\begin{aligned}
& {\left[\delta_{1}^{G}\left(\lambda_{1}\right), \delta_{1}^{G}\left(\lambda_{2}\right)\right]=\delta_{1}^{G}\left(\left[\lambda_{1}, \lambda_{2}\right]\right),} \\
& {\left[\delta_{2}^{G}\left(\lambda_{1}\right), \delta_{2}^{G}\left(\lambda_{2}\right)\right]=-\delta_{1}^{G}\left(\left[\lambda_{1}, \lambda_{2}\right]\right),} \\
& {\left[\delta_{1}^{G}\left(\lambda_{1}\right), \delta_{2}^{G}\left(\lambda_{2}\right)\right]=\delta_{2}^{G}\left(\left[\lambda_{1}, \lambda_{2}\right]\right),}
\end{aligned}
$$

leading to the $\mathrm{W}-\mathrm{Z}$ conditions:

$$
\begin{aligned}
& \delta_{1}^{G}\left(\lambda_{1}\right) a_{1}^{G}\left(\lambda_{2}\right)-\delta_{1}^{G}\left(\lambda_{2}\right) a_{1}^{G}\left(\lambda_{1}\right)=a_{1}^{G}\left(\left[\lambda_{1}, \lambda_{2}\right]\right), \\
& \delta_{2}^{G}\left(\lambda_{1}\right) a_{2}^{G}\left(\lambda_{2}\right)-\delta_{2}^{G}\left(\lambda_{2}\right) a_{2}^{G}\left(\lambda_{1}\right)=-a_{1}^{G}\left(\left[\lambda_{1}, \lambda_{2}\right]\right), \\
& \delta_{1}^{G}\left(\lambda_{1}\right) a_{2}^{G}\left(\lambda_{2}\right)-\delta_{2}^{G}\left(\lambda_{2}\right) a_{1}^{G}\left(\lambda_{1}\right)=a_{2}^{G}\left(\left[\lambda_{1}, \lambda_{2}\right]\right) .
\end{aligned}
$$

It can be shown [8, 13] that two non-equivalent local solutions of eqs.(17) are characterized by:

$$
\begin{aligned}
& a_{1}^{G}=0 \\
& a_{2}^{G} \neq 0
\end{aligned}
$$

and

$$
a_{2}^{G}= \pm i a_{1}^{G}
$$

The functional form of these solutions is given in [8]: they are local functionals of the connection $A_{\mu}$ on $S L(N, C)$ and of its derivatives. Projecting $A_{\mu}$ on $S U(N), a_{2}^{G}$, as derived from eq.(18), is the covariant anomaly, while $a_{1}^{G}$ in eq.(19) is the consistent anomaly. No differences arise for the $S O(2 n, C)$ sector.

The characterization of the consistent anomaly obtained by eq.(19) will be our guide in the construction of $\Gamma[A, E]$ : we are looking for a definition of $\operatorname{det}[D]$ compatible with eq.(19). We will also find a functional realization of eq.(18) that we will show to correspond to the modulus of the Weyl determinant.

\section{The two dimensional problem: algebraic and functional approach}

We can now apply the general properties discussed in the previous section to the twodimensional situation: some simplifications related to the peculiarity of $d=2$ take place, as we will see.

The first task is to realize the algebras eqs.(16) for $S L(N, C)$ and $S O(2, C)$. The general form of a $S L(N, C)$ Lie valued gauge connection is [14]:

$$
\hat{A}_{\mu}=\frac{i}{2}\left(\delta_{\mu}^{\nu}-i \frac{\epsilon_{\mu}^{\nu}}{\sqrt{g}}\right) V^{-1} \partial_{\nu} V+\frac{i}{2}\left(\delta_{\mu}^{\nu}+i \frac{\epsilon_{\mu}^{\nu}}{\sqrt{g}}\right) U^{-1} \partial_{\nu} U
$$


with $U$ and $V$ taking value in the $S L(N, C)$ group. The projection of $\hat{A}_{\mu}$ over $S U(N)$ is obtained within the restriction

$$
U^{\dagger}=V^{-1}
$$

that implies $\hat{A}_{\mu}^{\dagger}=\hat{A}_{\mu}$.

We define the action of $\delta_{1}^{G}$ and $\delta_{2}^{G}$ on $U$ and $V$ as:

$$
\begin{array}{lll}
\delta_{1}^{G}(\lambda) U & =-i U \lambda & \delta_{2}^{G}(\lambda) U=-U \lambda \\
\delta_{1}^{G}(\lambda) V=-i V \lambda & \delta_{2}^{G}(\lambda) V=V \lambda
\end{array}
$$

that leads to

$$
\begin{aligned}
\delta_{1}^{G}(\lambda) \hat{A}_{\mu} & =\hat{D}_{\mu} \lambda \\
\delta_{2}^{G}(\lambda) \hat{A}_{\mu} & =\epsilon_{\mu}^{\nu} \hat{D}_{\nu} \lambda
\end{aligned}
$$

with

$$
\hat{D}_{\mu} \lambda=\partial_{\mu} \lambda+i\left[\lambda, \hat{A}_{\mu}\right]
$$

One can easily show that $\delta_{1}^{G}(\lambda)$ and $\delta_{2}^{G}(\lambda)$ defined by eqs.(23) represent the algebra eq.(16). Following the general procedure based on the descent equation formalism [1], developed in [8, 9], we get the covariant solution eq.(18):

$$
\begin{aligned}
& a_{1}^{G}(\lambda)=0 \\
& a_{2}^{G}(\lambda)=\frac{1}{4 \pi} \int d^{2} x \sqrt{g} \operatorname{tr}\left[\left(\frac{1}{\sqrt{g}} \epsilon^{\mu \nu} \partial_{\mu}\left(\hat{A}_{\nu}+\hat{A}_{\nu}^{\dagger}\right)+\frac{i}{\sqrt{g}} \epsilon^{\mu \nu}\left[\hat{A}_{\mu}, \hat{A}_{\nu}^{\dagger}\right]\right) \lambda\right] .
\end{aligned}
$$

In the same way the consistent solution eq.(19) is:

$$
\begin{aligned}
& a_{1}^{G}(\lambda)=i a_{2}^{G}(\lambda)=\frac{i}{4 \pi} \int d^{2} x \sqrt{g} \operatorname{tr}\left[\left(\frac{1}{\sqrt{g}} \epsilon^{\mu \nu} \partial_{\mu} \hat{A}_{\nu}-i \mathcal{D}_{\mu} \hat{A}^{\mu}\right) \lambda\right] \\
& a_{1}^{G}(\lambda)=-i a_{2}^{G}(\lambda)=-\frac{i}{4 \pi} \int d^{2} x \sqrt{g} \operatorname{tr}\left[\left(\frac{1}{\sqrt{g}} \epsilon^{\mu \nu} \partial_{\mu} \hat{A}_{\nu}^{\dagger}+i \mathcal{D}_{\mu} \hat{A}^{\dagger \mu}\right) \lambda\right] .
\end{aligned}
$$

where

$$
\mathcal{D}_{\mu} f^{\nu}=\hat{D}_{\mu} f^{\nu}+\Gamma_{\mu \rho}^{\nu} f^{\rho} .
$$

Under $S U(N)$ projection they reduce to the well known expressions for the covariant and the consistent anomalies. The Bose factor $\frac{1}{2}$ is a natural bonus of the canonical normalization of the Lie algebra valued symmetric polynomials we have used to derive the descent equations.

To obtain a basis for the representation of $S O(2, C)$ we do not need a complexification of the zwei-bein $E_{a}^{\mu}$ : the abelian character of the group simplifies the approach. We use the representation of the Weyl algebra:

$$
\begin{aligned}
& \sigma_{1}=\tilde{\sigma}_{1}=1, \\
& \sigma_{2}=-\tilde{\sigma}_{2}=i
\end{aligned}
$$


and we define

$$
\begin{aligned}
E_{\mu} & =E_{\mu}^{a} \tilde{\sigma}_{a} \\
e^{\mu} & =e_{a}^{\mu} \sigma_{a} \\
\Omega_{\mu} & =-\frac{1}{4} \Omega_{\mu a b} \tilde{\sigma}_{a} \sigma_{b} .
\end{aligned}
$$

An $S O(2, C)$ matrix $\Lambda$ admits the factorization

$$
\Lambda=R \hat{\Lambda}
$$

where $R \in S O(2, R)$ and

$$
\hat{\Lambda}=\left(\begin{array}{cc}
\cosh \phi & -i \sinh \phi \\
i \sinh \phi & \cosh \phi
\end{array}\right)
$$

The action of $\hat{\Lambda}$ is:

$$
\begin{aligned}
\hat{\Lambda} E_{\mu} & =\exp (\phi) E_{\mu} \\
\hat{\Lambda} e^{\mu} & =\exp (-\phi) e^{\mu} \\
\hat{\Lambda} \Omega_{\mu} & =\Omega_{\mu}+\frac{1}{2} \frac{\epsilon_{\mu}^{\nu}}{\sqrt{g}} \partial_{\nu} \phi
\end{aligned}
$$

It is clear that the set of fields

$$
\begin{aligned}
\hat{E}_{\mu} & =\exp (\phi) E_{\mu}^{a} \\
\hat{e}^{\mu} & =\exp (-\phi) e_{a}^{\mu} \\
\hat{\Omega}_{\mu} & =\Omega_{\mu}+\frac{1}{2} \frac{\epsilon_{\mu}^{\nu}}{\sqrt{g}} \partial_{\nu} \phi .
\end{aligned}
$$

now carries a representation of $S O(2, C)$ Lie algebra:

$$
\begin{aligned}
& \delta_{1}^{L}(\tau) \hat{E}_{\mu}=\tau^{a b} \hat{E}_{\mu}^{b} \\
& \delta_{2}^{L}(\tau) \hat{E}_{\mu}=i \tau^{a b} \hat{E}_{\mu}^{b}
\end{aligned}
$$

derived from

$$
\begin{aligned}
\delta_{1}^{L}(\tau) E_{\mu}^{a} & =\tau_{a b} E_{\mu}^{b} \\
\delta_{2}^{L}(\tau) \phi & =\frac{i}{2} \tau_{a b} \tilde{\sigma}_{a} \sigma_{b}
\end{aligned}
$$

being $\delta_{1}^{L}(\tau) \phi=0=\delta_{2}^{L}(\tau) E_{\mu}^{a}$.

Basically we have recognized that

$$
\text { Lie } S O(2, C) \simeq \operatorname{Lie}\left(S O(2, R) \otimes R_{+}\right)
$$


and we understand $R_{+}$as the group of the local dilatations of the orthogonal frame (conformal transformations). The projection over $S O(2, R)$ is obtained setting $\phi=0$.

Using the descent equation, that in this case is almost trivial due to the abelian character of the problem, we get:

$$
\begin{aligned}
a_{1}^{L}(\tau) & =0 \\
a_{2}^{L}(\tau) & =\frac{i}{24 \pi} \int d^{2} x \sqrt{g} R \tau
\end{aligned}
$$

and

$$
a_{1}^{L}(\tau)= \pm i a_{2}^{L}(\tau)= \pm \int d^{2} x \sqrt{g}\left[\frac{1}{48 \pi}\left(R \pm 2 \Delta_{g} \phi\right) \pm \frac{i}{12 \pi} \mathcal{D}_{\mu} \hat{\Omega}^{\mu}\right] \tau
$$

$R$ being the usual scalar curvature.

We remark again the factor $\frac{1}{2}$ between the covariant and the consistent solution and the coboundary

$$
\pm \frac{i}{12 \pi} \int d^{2} x \sqrt{g} \mathcal{D}_{\mu} \hat{\Omega}^{\mu} \tau
$$

that is essential to obtain $a_{1}= \pm i a_{2}$. The abelian character of $S O(2, R)$ makes somewhat questionable the difference between consistent and covariant Lorentz anomaly in $d=2$ : both are proportional to $R$ (apart from coboundary terms) and the definition 12 requires only the factor $\frac{1}{2}$. Nevertheless in the extended situation they belong to two different cohomology classes of $\delta^{L}=\delta_{1}^{L}+\delta_{2}^{L}$; as one could check no local term brings eq.(34) into eq.(35).

All those algebraic properties possess a precise counterpart once we try to derive a meaningful functional from the naive definition eq.(6) of $\Gamma[A, E]$.

Let us turn our attention to the Weyl operator $D$ : a very general way to construct the determinant of a (pseudo) differential operator of elliptic type is by means of the $\zeta$-function regularization [4].

Under some assumptions we can define:

$$
\begin{aligned}
\zeta(s ; \mathcal{A}) & =\operatorname{Tr}\left[\mathcal{A}^{-s}\right]=\int d^{2} x \sqrt{g} \operatorname{tr}[K(x, x ; s)] \\
\operatorname{det}[\mathcal{A}] & =\exp \left[-\frac{d}{d s} \zeta(s ; \mathcal{A})\right]_{s=0}
\end{aligned}
$$

where $K(x, y ; s)$ is the kernel of the complex power of the elliptic operator $\mathcal{A}$ 15

$$
<x\left|\mathcal{A}^{-s}\right| y>=K(x, y ; s)
$$

and $\operatorname{Tr}$ is understood as an operatorial trace while $t r$ is a matrix trace.

$K(x, y ; s)$ is related to the more usual heat-kernel $H(x, y ; t)$ 16] by:

$$
K(x, y ; s)=\frac{1}{\Gamma(s)} \int_{0}^{\infty} d t t^{s-1} H(x, y ; t)
$$

Unfortunately we cannot apply directly the $\zeta$-function machinery to the Weyl operator: we fail because there is no way to define its complex power eq. (38) for the lack of a ray of 
minimal growth [15]. This is the analytic counterpart of the bad definition of the eigenvalue problem, discussed in sect.1.

To overcome this difficulty, we go back to eq.(6) and we introduce an isomorphism $D^{*}$

$$
D^{*}: \Gamma\left(S_{-}\right)_{M} \rightarrow \Gamma\left(S_{+}\right)_{M} ;
$$

then we define

$$
\operatorname{det}[D]=\operatorname{det}\left[D^{*} D\right] .
$$

The operator $D^{*} D$ admits a well defined eigenvalue problem, even if the covariance properties are, a priori, lost. Our task is to find such a $D^{*}$ to recover, under $S L(N, C)$ and $S O(2, C)$ variations, the consistent and the covariant solutions eq.(18), eq.(19). A good candidate for $D^{*}$ is

$$
D^{*}\left(r_{1}, r_{2}\right)=i \hat{\sigma}_{a} \hat{e}_{a}^{\mu}\left[\partial_{\mu}+\frac{1}{4}\left(1-r_{1}\right) \hat{\Omega}_{\mu}+i r_{2} \hat{A}^{\dagger}{ }_{\mu}\right]
$$

with $r_{1}, r_{2} \in R$. Taking into account our choice of the Weyl matrices we get for $r_{1}=0$, $r_{2}=1$ :

$$
D^{*}=D^{\dagger}
$$

and

$$
\operatorname{det}\left[D^{*}(0,1) D\right]=\operatorname{det}\left[D^{\dagger} D\right]=|\operatorname{det}[D]|^{2}
$$

In this case we lose the phase of the determinant on which the unextended anomaly relies [3].

One can prove that $D^{*}\left(r_{1}, r_{2}\right) D$ possesses a ray of minimal growth and the $\zeta$-function technique is perfectly viable. Defining

$$
\Gamma^{\left(r_{1}, r_{2}\right)}\left[\hat{E} ; \hat{A}, \hat{A}^{\dagger}\right]=\frac{1}{k\left(r_{1}, r_{2}\right)}\left[\frac{d}{d s} \zeta\left(s ; D^{*} D\right)\right]_{s=0}
$$

(the choice of the normalization $k\left(r_{1}, r_{2}\right)$ will be clarified in the following) and the anomalies

$$
\begin{array}{ll}
a_{1}^{G}(\lambda)=\delta_{1}^{G}(\lambda) \Gamma^{\left(r_{1}, r_{2}\right)} & a_{1}^{L}(\tau)=\delta_{1}^{L}(\tau) \Gamma^{\left(r_{1}, r_{2}\right)} \\
a_{2}^{G}(\lambda)=\delta_{2}^{G}(\lambda) \Gamma^{\left(r_{1}, r_{2}\right)} & a_{2}^{L}(\tau)=\delta_{2}^{L}(\tau) \Gamma^{\left(r_{1}, r_{2}\right)}
\end{array}
$$

we can find the values of $r_{1}, r_{2}$ satisfying eqs. (18) and (19) with local $a_{1,2}^{(G, L)}$.

To compute the variations of $\Gamma^{\left(r_{1}, r_{2}\right)}$ we need the transformation properties of $D^{*} D$ under $S L(N, C)$ and $S O(2, C)$ : using eq.(23) and eq.(32) one obtains:

$$
\begin{aligned}
\delta_{1}^{G}(\lambda)\left(D^{*} D\right) & =i\left(D^{*} D\right) \lambda-i r_{2} \lambda\left(D^{*} D\right)-\left(1-r_{2}\right)\left(D^{*} \lambda D\right)+ \\
& +i\left(1-r_{2}\right) r_{2} \tilde{\sigma}_{a} \hat{e}_{a}^{\mu}\left[\lambda, \hat{A}^{\dagger}{ }_{\mu}\right] D \\
\delta_{2}^{G}(\lambda)\left(D^{*} D\right) & =\left(D^{*} D\right) \lambda+r_{2} \lambda\left(D^{*} D\right)-\left(1+r_{2}\right)\left(D^{*} \lambda D\right)+ \\
& +i\left(1-r_{2}\right) r_{2} \tilde{\sigma}_{a} \hat{e}_{a}^{\mu}\left[\lambda, \hat{A}^{\dagger} \mu\right]
\end{aligned}
$$




$$
\begin{gathered}
\delta_{1}^{L}(\tau)\left(D^{*} D\right)=\left(1+r_{1}\right) \tau\left(D^{*} D\right)+\left(D^{*} D\right) \tau-r_{1}\left(D^{*} \tau D\right), \\
\delta_{2}^{L}(\tau)\left(D^{*} D\right)=-i\left(1+r_{1}\right) \tau\left(D^{*} D\right)-i\left(D^{*} D\right) \tau+\left(r_{1}-2\right)\left(D^{*} \tau D\right) .
\end{gathered}
$$

The trace properties of the kernel eq.(38) [15] allow us to write:

$$
\begin{aligned}
a_{1}^{G}(\lambda)= & \frac{1}{k} \frac{i}{4 \pi} \int d^{2} x \sqrt{g} \operatorname{tr}\left[\left(H_{1}\left(r_{1}, r_{2}\right)-\tilde{H}_{1}\left(r_{1}, r_{2}\right)\right) \lambda\right]\left(1-r_{2}\right)+ \\
+ & \frac{1}{k} \frac{i}{4 \pi}\left(1-r_{2}\right) r_{2} \int d^{2} x \sqrt{g} \frac{d}{d s}\left[s \operatorname{Tr}\left\{\tilde{\sigma}_{a} \hat{e}_{a}^{\mu}\left[\hat{A}^{\dagger} \mu, i \lambda\right] D\left(D^{*} D\right)^{-s-1}\right\}\right]_{s=0}, \\
a_{2}^{G}(\lambda)= & \frac{1}{k} \frac{i}{4 \pi} \int d^{2} x \sqrt{g} \operatorname{tr}\left[\left(H_{1}\left(r_{1}, r_{2}\right)-\tilde{H}_{1}\left(r_{1}, r_{2}\right)\right) \lambda\right]\left(1+r_{2}\right)+ \\
+ & \frac{1}{k} \frac{i}{4 \pi}\left(1-r_{2}\right) r_{2} \int d^{2} x \sqrt{g} \frac{d}{d s}\left[s \operatorname{Tr}\left\{\tilde{\sigma}_{a} \hat{e}_{a}^{\mu}\left[\hat{A}^{\dagger}{ }_{\mu}, \lambda\right] D\left(D^{*} D\right)^{-s-1}\right\}\right]_{s=0}, \\
& a_{1}^{L}(\tau)=\frac{1}{k} \frac{1}{4 \pi} \int d^{2} x \sqrt{g} \operatorname{tr}\left[\left(H_{1}\left(r_{1}, r_{2}\right)-\tilde{H}_{1}\left(r_{1}, r_{2}\right)\right) \tau\right] r_{1} \\
a_{2}^{L}(\tau)= & \frac{1}{k} \frac{i}{4 \pi} \int d^{2} x \sqrt{g} \operatorname{tr}\left[\left(H_{1}\left(r_{1}, r_{2}\right)-\tilde{H}_{1}\left(r_{1}, r_{2}\right)\right) r_{1}+4\left(H_{1}+\tilde{H}_{1}\right)\right] \tau,
\end{aligned}
$$

$H\left(r_{1}, r_{2}\right)$ and $\tilde{H}\left(r_{1}, r_{2}\right)$ being the first coefficients of the expansion of the heat-kernel eq.(37) for $t \rightarrow 0$ [16], generated respectively by the operators $D^{*} D$ and $D D^{*}$. Generalizing the technique developed in [6] to our case (a deformation of the operator $D^{\dagger} D$ ) we compute the coefficients $H_{1}$ and $\tilde{H}_{1}$ :

$$
\begin{aligned}
H_{1}\left(r_{1}, r_{2}\right) & =\left[-\frac{1}{24}-\frac{1}{8} r_{1}\right]\left(R+2 \Delta_{g} \phi\right)-\frac{i}{2} r_{1} \mathcal{D}_{\mu} \hat{\Omega}^{\mu}- \\
& -\frac{1}{2}\left[\frac{\epsilon^{\mu \nu}}{\sqrt{g}} \partial_{\mu}\left(\hat{A}_{\nu}+r_{2} \hat{A}_{\nu}^{\dagger}\right)+2 i r_{2} \frac{\epsilon^{\mu \nu}}{\sqrt{g}} \hat{A}_{\mu}^{\dagger} \hat{A}_{\nu}-i \mathcal{D}^{\mu}\left(\hat{A}_{\mu}-r_{2} \hat{A}_{\mu}^{\dagger}\right)\right] \\
\tilde{H}_{1}\left(r_{1}, r_{2}\right) & =\left[-\frac{1}{24}+\frac{1}{8} r_{1}\right]\left(R+2 \Delta_{g} \phi\right)+\frac{i}{2} r_{1} \mathcal{D}_{\mu} \hat{\Omega}^{\mu}- \\
& -\frac{1}{2}\left[-\frac{\epsilon^{\mu \nu}}{\sqrt{g}} \partial_{\mu}\left(\hat{A}_{\nu}+r_{2} \hat{A}_{\nu}^{\dagger}\right)+2 i r_{2} \frac{\epsilon^{\mu \nu}}{\sqrt{g}} \hat{A}_{\mu}^{\dagger} \hat{A}_{\nu}+i \mathcal{D}^{\mu}\left(\hat{A}_{\mu}-r_{2} \hat{A}_{\mu}^{\dagger}\right)\right] .
\end{aligned}
$$

We remark that the definition eq.(44) automatically implements the invariance under diffeomorphism: one can easily check that

$$
\delta^{D}(\xi)\left(D^{*} D\right)=\left[\xi^{\mu} \partial_{\mu}, D^{*} D\right]
$$

the trace properties of the kernel eq.(37) implies that this type of transformations does not change the $\zeta$-function determinant.

Let us now look for the covariant and the consistent solution. 


\section{The covariant determinant and its $\mathrm{W}-\mathrm{Z}-\mathrm{W}$ term}

A local expression for $a_{2}^{G}(\lambda)$ and $a_{2}^{L}(\tau)$, compatible with

$$
a_{1}^{G}(\lambda)=0=a_{1}^{L}(\tau),
$$

is obtained by choosing

$$
\begin{aligned}
& r_{1}=0 \\
& r_{2}=1
\end{aligned}
$$

that corresponds to take $D^{*}=D^{\dagger}$; the normalization $k$ is fixed to $\frac{1}{2}$ to recover:

$$
\Gamma^{(0,1)}\left[\hat{E} ; \hat{A}, \hat{A}^{\dagger}\right]=-\log |\operatorname{det}[D]|
$$

The explicit form of $H_{1}$ and $\tilde{H}_{1}$ leads to the anomalies:

$$
\begin{gathered}
a_{2}^{G}(\lambda)=\frac{1}{4 \pi} \int d^{2} x \sqrt{g} \operatorname{tr}\left[\left\{\frac{\epsilon^{\mu \nu}}{\sqrt{g}} \partial_{\mu}\left(\hat{A}_{\nu}+\hat{A}_{\nu}^{\dagger}\right)+\right.\right. \\
\left.\left.+i \frac{\epsilon^{\mu \nu}}{\sqrt{g}}\left[\hat{A}_{\mu} \hat{A}_{\nu}^{\dagger}\right]-i \mathcal{D}^{\mu}\left(\hat{A}_{\mu}-\hat{A}_{\mu}^{\dagger}\right)\right\} \lambda\right] \\
a_{2}^{L}(\tau)=\frac{i}{24 \pi} \int d^{2} x \sqrt{g}\left(R+2 \Delta_{g} \phi\right) \tau,
\end{gathered}
$$

that for $\hat{A}_{\mu}=\hat{A}_{\mu}^{\dagger}$ and $\phi=0$ become the usual covariant gauge and Lorentz anomalies:

$$
\begin{aligned}
& a_{\text {cov }}^{G}(\lambda)=\frac{1}{4 \pi} \int d^{2} x \sqrt{g} \operatorname{tr}\left[\left(\frac{\epsilon^{\mu \nu}}{\sqrt{g}} F_{\mu \nu}\right) \lambda\right] \\
& a_{c o v}^{L}(\tau)=\frac{i}{24 \pi} \int d^{2} x \sqrt{g} R \tau
\end{aligned}
$$

The next step is to compute eq.(58): a first semplification derives from the fact that $\hat{E}_{\mu}^{a}$ is the conformal transformed of $E_{\mu}^{a}$, giving us the possibility of working with the original zwei-bein and, at the end, to recover the extended result with a local dilatation. A second observation concerns the diffeomorphism invariance of the determinant itself: we can choose a convenient coordinate system to develop our calculations.

Locally any two-dimensional Riemannian manifold admits a coordinate system [17] in which the metric tensor has the form

$$
g_{\mu \nu}=\exp (4 \alpha) \delta_{\mu \nu}
$$

leading to the zwei-bein

$$
e_{a}^{\mu}=\exp (-2 \alpha) \delta_{b}^{\mu}\left(\delta_{a b} \cos (2 \beta)-\epsilon_{a b} \sin (2 \beta)\right) .
$$


Disregarding problems of globality, we assume everywhere the validity of this parameterization, obtaining a simple expression for the spin-connection and the scalar curvature:

$$
\begin{aligned}
\Omega_{\mu} & =\partial_{\mu} \beta+\epsilon_{\mu \nu} \partial_{\nu} \alpha \\
R & =-4 \frac{1}{\sqrt{g}} \partial_{\mu} \partial_{\mu} \alpha .
\end{aligned}
$$

In these coordinates $D^{\dagger} D$ can be written as

$$
\begin{aligned}
D^{\dagger} D & =i \tilde{\sigma}_{\mu} \exp [-3 \alpha-i \beta]\left(\partial_{\mu}+i \hat{A}_{\mu}^{\dagger}\right) \exp [-2 \alpha] . \\
& \cdot i \sigma_{\nu}\left(\partial_{\nu}+i \hat{A}_{\nu}\right) \exp [\alpha+i \beta] .
\end{aligned}
$$

It is not difficult to find the infinitesimal variation of $\operatorname{det}\left[D^{\dagger} D\right]$ for the transformations:

$$
\begin{aligned}
& \alpha \rightarrow \alpha-\varepsilon \alpha \\
& \beta \rightarrow \beta-\varepsilon \beta
\end{aligned}
$$

with $\varepsilon \rightarrow 0$ and to iterate this change driving $\alpha$ and $\beta$ to zero:

$$
\begin{aligned}
\operatorname{det}\left[D^{\dagger} D\right] & =\exp \left[\Gamma_{1}\right] \operatorname{det}\left[D_{2}^{\dagger} D_{2}\right] \\
D_{2} & =i \sigma_{\mu}\left(\partial_{\mu}+i \hat{A}_{\mu}\right) .
\end{aligned}
$$

One can easily verify that $\Gamma_{1}$ actually is only a functional of $\alpha$ and, eventually, of $\hat{A}_{\mu}$ and $\hat{A}_{\mu}^{\dagger}$ : this fact reflects its invariance under $S O(2, R)$ Lorentz transformations (independence from the local orthogonal frame, that is contained in $\beta$, as it is evident from eq.(63)). In terms of de-Witt coefficients, $\Gamma_{1}$ is given [5] by:

$$
\begin{aligned}
\Gamma_{1} & =\int_{0}^{1} d y \frac{d \Gamma_{1}}{d y}(y) \\
\frac{d \Gamma_{1}}{d y}(y) & =\frac{1}{8 \pi} \int d^{2} x \sqrt{g(y)}\left[H_{1}\left(D^{\dagger}(y) D(y)\right)+\tilde{H}_{1}\left(D(y) D^{\dagger}(y)\right)\right] 2 \alpha
\end{aligned}
$$

where

$$
\sqrt{g(y)}=\exp [4 \alpha(1-y)]
$$

and $D^{\dagger}(y) D(y), D(y) D^{\dagger}(y)$ are obtained from eq.(65) with the substitution

$$
\begin{aligned}
& \alpha \rightarrow \alpha(1-y), \\
& \beta \rightarrow \beta(1-y) .
\end{aligned}
$$

The heat-kernel coefficients are derived from eq.(54) and eq.(55):

$$
\Gamma_{1}[\alpha]=\frac{1}{24 \pi} \int d^{2} x \int_{0}^{1} d y(1-y) 4\left(\partial_{\mu} \partial_{\mu} \alpha\right) \alpha,
$$


that in covariant form is:

$$
\Gamma_{1}=\frac{1}{192 \pi} \int d^{2} x \sqrt{g} d^{2} z \sqrt{g} R(x) \Delta_{g}^{-1}(x, z) R(z),
$$

$\Delta_{g}^{-1}(x, z)$ being the kernel of the inverse Beltrami-Laplace operator.

We still have to compute

$$
\operatorname{det}\left[D_{2}^{\dagger} D_{2}\right]=\operatorname{det}[-\Delta] \exp -\Gamma_{2}\left[\hat{A}, \hat{A}^{\dagger}\right]
$$

$\operatorname{det}[-\Delta]$ giving the natural normalization to the free case (no gauge or gravitational background).

At this point we observe that

$$
\begin{aligned}
& \frac{1}{2} \sigma_{\mu}\left(\delta_{\mu \nu}-i \epsilon_{\mu \nu}\right)=\sigma_{\nu} \\
& \frac{1}{2} \tilde{\sigma}_{\mu}\left(\delta_{\mu \nu}+i \epsilon_{\mu \nu}\right)=\tilde{\sigma}_{\nu}
\end{aligned}
$$

while

$$
\frac{1}{2} \sigma_{\mu}\left(\delta_{\mu \nu}+i \epsilon_{\mu \nu}\right)=\frac{1}{2} \tilde{\sigma}_{\mu}\left(\delta_{\mu \nu}-i \epsilon_{\mu \nu}\right)=0
$$

recalling the form of the $\hat{A}_{\mu}$ connection given in eq.(20) we get:

$$
\begin{aligned}
D_{2}^{\dagger} D_{2} & =i \tilde{\sigma}_{\mu}\left[\partial_{\mu}-V^{\dagger} \partial_{\mu}\left(V^{\dagger}\right)^{-1}\right] i \sigma_{\nu}\left[\partial_{\nu}-V^{-1} \partial_{\nu} V\right] \\
& =V^{\dagger}\left(i \tilde{\sigma}_{\mu} \partial_{\mu}\right)\left(V V^{\dagger}\right)^{-1}\left(i \sigma_{\nu} \partial_{\nu}\right) V .
\end{aligned}
$$

If we define an interpolating matrix $V(r)$ with the property

$$
\begin{aligned}
& V(0)=1 \\
& V(1)=V
\end{aligned}
$$

we can express, by means of the same decoupling technique used on eq.(68), $\Gamma_{2}$ as:

$$
\begin{aligned}
\Gamma_{2}\left[V, V^{\dagger}\right] & =\int_{0}^{1} d r \frac{d \Gamma_{2}}{d r}\left[r ; V, V^{\dagger}\right] \\
\frac{d \Gamma_{2}}{d r}\left[r ; V, V^{\dagger}\right] & =\frac{1}{4 \pi} \int d^{2} x \operatorname{tr}\left[( H _ { 1 } ( r ) - \tilde { H } _ { 1 } ( r ) ) \left(V^{-1}(r) \partial_{r} V(r)-\right.\right. \\
& \left.\left.-V^{\dagger}(r) \partial_{r}\left(V^{\dagger}\right)^{-1}(r)\right)\right]
\end{aligned}
$$

where $H_{1}(r)$ and $\tilde{H}_{1}(r)$ are again derived from eq.(54) and eq.(55), taking the pure gauge part. They give:

$$
\begin{aligned}
H_{1}(r)-\tilde{H}_{1}(r) & =-\frac{1}{2}\left(\delta_{\mu \nu}+i \epsilon_{\mu \nu}\right) \partial_{\mu}\left[V^{\dagger} \partial_{\nu}\left(V^{\dagger}\right)^{-1}\right](r)+\frac{1}{2}\left(\delta_{\mu \nu}-i \epsilon_{\mu \nu}\right) \partial_{\mu}\left[V \partial_{\nu} V^{-1}\right](r)- \\
& -\frac{1}{2}\left(\delta_{\mu \nu}-i \epsilon_{\mu \nu}\right)\left[V^{-1} \partial_{\mu} V V^{\dagger} \partial_{\nu}\left(V^{\dagger}\right)^{-1}+V^{\dagger} \partial_{\mu}\left(V^{\dagger}\right)^{-1} V^{-1} \partial_{\nu} V\right]
\end{aligned}
$$


It is straightforward to compute the trace on eq.(75) and to obtain:

$$
\begin{aligned}
\Gamma_{2}\left[V, V^{\dagger}\right]=\hat{\Gamma}\left[V V^{\dagger}\right] & =\frac{1}{8 \pi} \int d^{2} x \operatorname{tr}\left[\partial_{\mu}\left(V V^{\dagger}\right) \partial_{\mu}\left(V V^{\dagger}\right)^{-1}\right]+S_{W Z W}\left[V V^{\dagger}\right] \\
S_{W Z W}[V] & =\frac{1}{4 \pi} \int_{0}^{1} d r \int d^{2} x \epsilon_{\mu \nu}\left[V^{-1}\left(\partial_{\mu} V\right) V^{-1}\left(\partial_{\nu} V\right) V^{-1}\left(\partial_{r} V\right)\right] .
\end{aligned}
$$

Taking eq.(77) in its coordinate invariant form and performing on eq.(71) a local dilatation, we can write the extended $D^{\dagger} D$ determinant (normalized to the free case):

$$
\begin{gathered}
\operatorname{det}\left[D^{\dagger} D\right]=\exp -\Gamma\left[\hat{E} ; \hat{A}, \hat{A}^{\dagger}\right] \\
\Gamma\left[\hat{E} ; \hat{A}, \hat{A}^{\dagger}\right]=\frac{1}{192 \pi} \int d^{2} x \sqrt{g} d^{2} z \sqrt{g} R(x) \Delta_{g}^{-1}(x, z) R(z)+ \\
+\frac{1}{48 \pi} \int d^{2} x \sqrt{g}\left[R \phi+\phi \Delta_{g} \phi\right]+\frac{1}{8 \pi} \int d^{2} x \sqrt{g} \operatorname{tr}\left[\left(V V^{\dagger}\right)^{-1} \Delta_{g}\left(V V^{\dagger}\right)\right]+ \\
+S_{W Z W}\left[V V^{\dagger}\right] .
\end{gathered}
$$

We notice that the extra-piece depending on $\phi$ generates a Liouville action: $\phi$ appears as a dilaton field.

Let us turn our attention to the gauge field part of eq.(78): we recall that $\delta_{2}^{G}$ generates transformations belonging to $S L(N, C) / S U(N)$. A finite transformation of this type acts on $V$ and $U$ as:

$$
\begin{aligned}
V & \rightarrow V \hat{h}, \\
U & \rightarrow U \hat{h}^{-1},
\end{aligned}
$$

where $\hat{h} \in S L(N, C) / S U(N) . \Gamma\left[\hat{E} ; \hat{A}, \hat{A}^{\dagger}\right]$ depends only on the combination $V V^{\dagger}$, so the invariance of eq.(78) under $S U(N)$ transformations is evident while under eq.(79) we have:

$$
V V^{\dagger} \rightarrow V \hat{h}^{2} V^{\dagger}
$$

The well-known Polyakov-Weigmann formula 18

$$
\hat{\Gamma}[A B]=\hat{\Gamma}[A]+\hat{\Gamma}[B]+\int d^{2} x \sqrt{g}\left(g^{\mu \nu}+i \frac{\epsilon^{\mu \nu}}{\sqrt{g}}\right) \operatorname{tr}\left[\left(A^{-1} \partial_{\mu} A\right)\left(B \partial_{\nu} B\right)\right]
$$

allows us to express the effect of the transformation $\hat{h}$ through a covariant W-Z-W action [9]:

$$
\begin{gathered}
\hat{\Gamma}\left[V \hat{h}^{2} V^{\dagger}\right]=\hat{\Gamma}\left[V V^{\dagger}\right]+\Gamma_{W Z W}^{c o v}\left[\hat{h} ; \hat{A}, \hat{A}^{\dagger}\right] \\
\Gamma_{W Z W}^{c o v}\left[\hat{h} ; \hat{A}, \hat{A}^{\dagger}\right]=2 \hat{\Gamma}[\hat{h}]+\frac{1}{4 \pi} \int d^{2} x \sqrt{g}\left(g^{\mu \nu}+i \frac{\epsilon^{\mu \nu}}{\sqrt{g}}\right) \operatorname{tr}\left[\hat{A}_{\mu} \hat{A}_{\nu}^{\dagger}\right]
\end{gathered}
$$




$$
\begin{aligned}
& -\frac{i}{4 \pi} \int d^{2} x \sqrt{g}\left(g^{\mu \nu}+i \frac{\epsilon^{\mu \nu}}{\sqrt{g}}\right) \operatorname{tr}\left[\hat{h}^{-2} \hat{A}_{\mu} \hat{h}^{2} \hat{A}_{\nu}^{\dagger}\right] \\
& -\frac{i}{4 \pi} \int d^{2} x \sqrt{g}\left(g^{\mu \nu}+i \frac{\epsilon^{\mu \nu}}{\sqrt{g}}\right) \operatorname{tr}\left[\hat{A}_{\mu} \hat{h}^{2} \partial_{\nu} \hat{h}^{-2}+\hat{h}^{-2} \partial_{\mu} \hat{h}^{2} \hat{A}_{\nu}^{\dagger}\right] \\
& +\frac{1}{4 \pi} \int d^{2} x \sqrt{g}\left(g^{\mu \nu}+i \frac{\epsilon^{\mu \nu}}{\sqrt{g}}\right) \operatorname{tr}\left[\hat{h}^{2}\left(\partial_{\mu} \hat{h}^{-1}\right)\left(\partial_{\nu} \hat{h}^{-1}\right)\right] \\
& +\frac{1}{4 \pi} \int d^{2} x \sqrt{g} \operatorname{tr}\left[\hat{h}^{2} \Delta_{g} \hat{h}^{-2}-2 \hat{h} \Delta_{g} \hat{h}^{-1}\right] .
\end{aligned}
$$

$\Gamma_{W Z W}^{c o v}$ was obtained, in $d=2 n$ dimensions, using cohomological methods in [9]; this is an explicit computation, based on a functional representation of the relevant extended algebra. The projection over $S U(N)$ is performed by taking $V^{\dagger}=U^{-1}$. In this limit $\hat{\Gamma}\left[V V^{\dagger}\right]$ coincides with the usual expression of the logarithm of the Dirac operator, confirming the well known property [3] that

$$
\left|\operatorname{det}\left[D_{\text {Weyl }}\right]\right|=\operatorname{det}\left[D_{\text {Dirac }}\right]
$$

Nevertheless by means of an infinitesimal $S L(N, C) / S U(n)$ transformation we can recover the covariant anomaly as one could directly check in eq.(82) starting from

$$
\hat{h} \simeq 1+\lambda \text {. }
$$

\section{The consistent determinant}

Finally we find the correct second order operator, realizing a mapping

$$
\Gamma\left(S_{+}\right)_{M} \rightarrow \Gamma\left(S_{+}\right)_{M}
$$

whose $\zeta$-function determinant represents the determinant of the Weyl operator. In ref.[8], in the case of pure gravity coupling, we have found a whole one-parameter family of second order operators, relaxing the definition in eq.(41), that leads to the consistent condition eq.(19).

Now we strictly study operators of the form $D^{*} D$, where $D$ is the Weyl operator: in other words we want to find the correct isomorphism $D^{*}$ in presence of an additional non-abelian gauge background. We start from eqs.(50), (51), (52), (53), with $k=1$, and we try to fix $r_{1}$ and $r_{2}$ leading to:

$$
\begin{aligned}
& a_{2}^{G}(\lambda)=-i a_{1}^{G}(\lambda), \\
& a_{2}^{L}(\tau)=-i a_{1}^{L}(\tau) .
\end{aligned}
$$

One immediately realizes that $r_{2}=0$ gives a local expression for $a_{1}^{G}$ and $a_{2}^{G}$ obeying to eq. (83). To derive the correct value of $r_{1}$ we first switch off the gauge field on eqs.(52), (53): 
then using the explicit form of $H_{1}\left(r_{1}, 0\right)$ and $\tilde{H}_{1}\left(r_{1}, 0\right)$ we obtain:

$$
\begin{aligned}
& a_{1}^{L}(\tau)=\frac{1}{4 \pi} \int d^{2} x \sqrt{g}\left[-\frac{1}{4} r_{1}^{2}\left(R+2 \Delta_{g} \phi\right)-i r_{1}^{2} \mathcal{D}_{\mu} \hat{\Omega}^{\mu}\right] \tau \\
& a_{2}^{L}(\tau)=\frac{i}{4 \pi} \int d^{2} x \sqrt{g}\left[-\frac{1}{4} r_{1}^{2}\left(R+2 \Delta_{g} \phi\right)-i r_{1}^{2} \mathcal{D}_{\mu} \hat{\Omega}^{\mu}+\frac{1}{3}\left(R+2 \Delta_{g} \phi\right)\right] \tau
\end{aligned}
$$

forcing the equations:

$$
\begin{aligned}
\frac{1}{4} r_{1}^{2} & =\frac{1}{3}-\frac{1}{4} r_{1}^{2} \\
r_{1}^{2} & =-r_{1}^{2}
\end{aligned}
$$

that are clearly inconsistent. But we recall that eq.(84) must be understood up to coboundary terms, a fact that corresponds to the addition of local terms on the logarithm of the determinant

$$
\Gamma^{\left(r_{1}, 0\right)}\left[\hat{E} ; \hat{A}, \hat{A}^{\dagger}\right]=-\log \operatorname{det}\left[D^{*}\left(r_{1}, 0\right) D\right] .
$$

If we add to the $\zeta$-function calculation of $\Gamma^{\left(r_{1}, 0\right)}\left[\hat{E} ; \hat{A}, \hat{A}^{\dagger}\right]$ the local polynomial

$$
P[\hat{E}]=-\frac{1}{12 \pi} \int d^{2} x \sqrt{g} g^{\mu \nu} \hat{\Omega}_{\mu} \hat{\Omega}_{\nu}
$$

we have to consider its contribution to $a_{1}^{L}(\tau)$ and $a_{2}^{L}(\tau)$ that is:

$$
\begin{aligned}
\delta_{1}^{L}(\tau) P[\hat{E}] & =-\frac{1}{6 \pi} \int d^{2} x \sqrt{g} \mathcal{D}_{\mu} \hat{\Omega}^{\mu} \tau \\
\delta_{2}^{L}(\tau) P[\hat{E}] & =-\frac{i}{24 \pi} \int d^{2} x \sqrt{g}\left(R+2 \Delta_{g} \phi\right) \tau .
\end{aligned}
$$

These contributions change eq.(85) and eq.(86), giving a new system that replaces eq.(87):

$$
\begin{aligned}
\frac{1}{2} r_{1}^{2} & =\frac{1}{3}-\frac{1}{6} \\
r_{1}^{2}-\frac{2}{3} & =-r_{1}^{2} .
\end{aligned}
$$

The two equations are actually the same, leading to the values

$$
r_{1}= \pm \frac{\sqrt{3}}{3}
$$

that are fully consistent with the general solution of ref. [8]. The restoration of the gauge fields $\hat{A}_{\mu}$ and $\hat{A}_{\mu}^{\dagger}$ does not change anything: one can easily verify that their contributions to $a_{1}^{L}(\tau)$ and $a_{2}^{L}(\tau)$, for $r_{2}=0$, automatically satisfy eq.(84), so no new constraints arise from 
their presence. To get the consistent gauge and Lorentz anomalies we have only to compute eq.(51) and eq.(52) with $r_{1}=\frac{\sqrt{3}}{3}$ and $r_{2}=0$ :

$$
\begin{aligned}
& a_{1}^{G}(\lambda)=\frac{i}{4 \pi} \int d^{2} x \sqrt{g} \operatorname{tr}\left[\left(\frac{\epsilon^{\mu \nu}}{\sqrt{g}} \partial_{\mu} \hat{A}_{\nu}-i \mathcal{D}_{\mu} \hat{A}^{\mu}\right) \lambda\right] \\
& a_{1}^{L}(\tau)=-\frac{1}{48 \pi} \int d^{2} x \sqrt{g}\left[R+2 \Delta_{g} \phi+4 i \mathcal{D}_{\mu} \hat{\Omega}^{\mu}\right] \tau .
\end{aligned}
$$

For $\phi=0$ and $\hat{A}_{\mu}=A_{\mu}$, we recover the usual form (up to trivial cocycles), with the correct normalization.

The computation of $\Gamma^{\left(\frac{\sqrt{3}}{3}, 0\right)}\left[\hat{E} ; \hat{A}, \hat{A}^{\dagger}\right]$ is now straightforward: as in the previous section we choose the coordinate system eq.(63), where $D^{*} D$ looks like

$$
\begin{aligned}
D^{*} D & =\exp \left[\alpha\left(r_{1}-3\right)-i \beta\left(r_{1}+1\right)\right]\left(i \tilde{\sigma} \partial_{\mu}\right) \exp \left[-\alpha\left(r_{1}+2\right)+i \beta r_{1}\right] . \\
& \cdot D_{2} \exp [\alpha+i \beta] \\
D_{2} & =i \sigma_{\nu}\left[\partial_{\nu}-V^{-1} \partial_{\nu} V\right]
\end{aligned}
$$

we do not exhibit the explicit calculation, similar to the one described in the previous section, that leads to

$$
\begin{gathered}
\Gamma^{\left(\frac{\sqrt{3}}{3}, 0\right)}=\hat{\Gamma}^{\left(\frac{\sqrt{3}}{3}, 0\right)}-\log \operatorname{det}\left[\left(i \tilde{\sigma}_{\mu} \partial_{\mu}\right) D_{2}\right] \\
\hat{\Gamma}^{\left(\frac{\sqrt{3}}{3}, 0\right)=} \frac{1}{192 \pi} \int d^{2} x \sqrt{g} d^{2} z \sqrt{g}\left[R(x) \Delta_{g}^{-1}(x, z) R(z)+i R(x) \Delta_{g}^{-1}(x, z) \frac{1}{\sqrt{g}} \partial_{\mu}\left(\sqrt{g} \Omega^{\mu}(z)\right)\right]- \\
-\frac{1}{24 \pi} \int d^{2} x \sqrt{g} \Omega_{\mu} \Omega^{\mu}
\end{gathered}
$$

where we have taken $\phi=0$ and we have expressed $\hat{\Gamma}^{\left(\frac{\sqrt{3}}{3}, 0\right)}$ in its diffeo-invariant form. The calculation of

$$
\hat{\Gamma}_{2}=-\log \operatorname{det}\left[\left(i \tilde{\sigma}_{\mu} \partial_{\mu}\right) D_{2}\right],
$$

can be directly obtained from eq.(77): we notice that the two determinants are strictly the same if we put $V^{\dagger}=11$ in eq.(73), so that

$$
\hat{\Gamma}_{2}=\hat{\Gamma}[V]
$$

the normalization to the free case is always understood. Summing the two different contributions

$$
\Gamma^{\left(\frac{\sqrt{3}}{3}, 0\right)}=\hat{\Gamma}^{\left(\frac{\sqrt{3}}{3}, 0\right)}+\hat{\Gamma}[V]
$$

we recognize the non-abelian generalization of the classical Leutwyler's result [7], with a different choice of the local term in the gravitational part. The Weyl determinant is obtained 
as the $\zeta$-function determinant of the second order operator (we make the projection on $S U(N)$ and $S L(2, R))$

$$
D^{*} D=i \tilde{\sigma}_{a} e_{a}^{\mu}\left[\partial_{\mu}+\left(1-\frac{\sqrt{3}}{3}\right) \Omega_{\mu}\right] i \sigma_{b} e_{b}^{\nu}\left[\partial_{\nu}+i A_{\nu}+\Omega_{\nu}\right]
$$

giving an explicit diffeo-invariant result. If we switch off the zwei-bein field, we recover the well-known form of the two-dimensional non-abelian gauge determinant [14]. The usual $\mathrm{W}-\mathrm{Z}-\mathrm{W}$ term is easily obtained by means of a $S U(N)$ gauge transformation

$$
V \rightarrow h V
$$

we notice that a coset trasformation, $\hat{h} \in S L(N, C) / S U(N)$, leads to the same action for the $\mathrm{W}-\mathrm{Z}-\mathrm{W}$ field. The characterization we have given of the consistent determinant, eq.(19), holds also for finite variations:

$$
\begin{array}{ll}
h: & \hat{\Gamma}[V] \rightarrow \hat{\Gamma}[V]+\Gamma_{W Z W}^{c o n}[h] \\
\hat{h}: & \hat{\Gamma}[V] \rightarrow \hat{\Gamma}[V]+\Gamma_{W Z W}^{c o n}[\hat{h}]
\end{array}
$$

\section{Conclusions}

We have obtained in $d=2$, by characterizing the consistent and the covariant anomalies as different solutions of an extended cohomological problem, two second order operators: using $\zeta$-function regularization they produce, respectively, the covariant and the consistent Lorentz and gauge anomalies. We have computed their determinants and the W-Z-W terms linked to the gauge part.

The "consistent" determinant is the non-abelian generalization of the one derived by Leutwyler in [7], as a consequence of a completely different procedure. If we switch-off the gauge field, the operator in eq.(100) is a particular case of the one-parameter family of operators, obtained in [ 8$]$, that led us to the same Weyl determinant (up to coboundary terms). At least to our knowledge, in the case of curved background, it is the first time that the Weyl determinant has been computed as the $\zeta$-function determinant of some operator.

The "covariant" determinant is essentially the modulus of the Dirac one: nevertheless working with a $S L(N, C)$ gauge group, this functional is not invariant under $S L(N, C) / S U(N)$ transformations. The coset action produces the covariant anomaly in the infinitesimal case, and, for finite transformations, the covariant W-Z-W term, derived in [9] by cohomological methods.

I would like to thank Prof. Antonio Bassetto for reading the manuscript and for many useful comments. I am also grateful to Dott. Pietro Donatis for having read the manuscript. 


\section{References}

[1] B. Zumino, Y-S. Wu, A. Zee: Nucl. Phys. B239 (1984), 477 ;

L. Bonora, P. Cotta-Ramusino: Comm. Math. Phys. $\underline{87}$ (1983), 589

[2] M.F. Atiyah, I.M. Singer: Proc.Natl.Acad.Sci. USA $\underline{81}$ (1984), 2597

[3] L. Alvarez-Gaumè, E. Witten: Nucl. Phys. B234 (1984), 269

[4] S. Hawking: Comm. Math. Phys. $\underline{55}$ (1977), 133

[5] R.E. Gamboa-Saravi, M.A. Muschietti, F. Schaposnik, J.E. Solomin: Ann. Phys. 157 (1984), 360

[6] H. Leutwyler, S. Mallik: Z. Phys. C $\underline{37}$ (1986), 205

[7] H. Leutwyler: Phys. Lett. 153B (1985), 65

[8] L. Griguolo: Covariant anomalies and functional determinants, hep-th 9312044. To be published in Fortschritte der Physik

[9] J. Manes, R. Stora, B. Zumino: Comm. Math. Phys. 102 (1985), 157

[10] H.Leutwyler: Helv. Phys. Acta $\underline{59}$ (1986), 201

[11] F. Langouche, T. Schucker, R. Stora: Phys. Lett. 145B (1984), 342

[12] W. Bardeen, B. Zumino: Nucl. Phys. B244 (1984), 421

[13] A. Bassetto, P. Giacconi, L. Griguolo, R. Soldati: Phys. Lett. 251B (1990), 266

[14] K.D. Rothe, Nucl. Phys. B269 (1986), 269

[15] R.T. Seeley: Ann. Math. Soc. Proc. Symp. Pure Math.10 (1967), 288

[16] P.B. Gilkey: The index theorem and the heat-equation. Boston Publish of perish (1974)

[17] N.J. Hicks: Notes on differential geometry, D. Van Nostrand Company (1965)

[18] A.M. Polyakov, P.B. Wiegmann: Phys. Lett. 131B (1983), 121 